\title{
Tätigkeitsvorausschau 2013 des EDSB
}

Am 18.01.2013 hat der Europäische Datenschutzbeauftragte (EDSB) sein Arbeitsprogramm 2013 im Bereich der Beratung zu Rechtsetzungsvorschlägen veröffentlicht, eingeschlossen sein strategisches Planungsdokument, die Tätigkeitsvorausschau. Der EDSB identifiziert in diesem Dokument Themen von strategischer Bedeutung, die die Eckpfeiler seiner Beratungsarbeit für 2013 bilden werden.

Peter Hustinx, EDSB: „Wir leben in einer technisch vermittelten Welt, die sich ständig weiterentwickelt Die Sichtbarkeit und Relevanz des Datenschutzes ist somit größer als je zuvor. Die Notwendigkeit, die Auswirkungen von Gesetzesvorschläge auf die Privatsphäre und den Datenschutz zu berücksichtigen, wird in allen Politikbereichen der EU unumgänglich und führt zu einer Zunahme neuer Politikbereiche, mit denen wir uns auseinandersetzen müssen. Es wird zunehmend erkennbar, dass das Grundrecht auf Datenschutz nicht allein im Datenschutzrecht reguliert werden kann, sondern dass viele andere Politikbereiche den Datenschutz berücksichtigen müssen.“

Da sich der EDSB mit wachsenden Herausforderungen und Aktivitäten in Zeiten knapper Haushaltsmittel konfrontiert sieht, umreißt die Tätigkeitsvorausschau eine Strategie und Handlungen, die der Arbeit des EDSB im Bereich des Datenschutzes maximale Wirkung auf EU-Ebene gestattet und deren Effizienz erhöht, indem er selektiver vorgeht und bestmöglichsten Gebrauch von Ressourcen macht.

Die Hauptbereiche von strategischer Bedeutung, die die Basis der Beratungsarbeit des EDSB für 2013 darstellen, umfassen:

- Einen neuen Rechtsrahmen für Datenschutz,

- Technische Entwicklungen und die Digitale Agenda,

- Weiterentwicklung des Raums der Freiheit, Sicherheit und Justiz,

- Reform des Finanzsektors, und

- eHealth.

Weiterhin wird der EDSB es erwägen, Stellungnahmen zur Integration von Datenschutz in anderen Politikbereichen der EU vorzulegen, so wie Wettbewerb und Handel.

Die laufenden Arbeiten an einem der umfassendsten Gesetzgebungsverfahren der letzten Jahre - den beiden Vorschlägen zur Reform des EU-Datenschutzrahmens - ist Gegenstand heftiger Spekulationen und Interessen für Akteure auf nationaler, europäischer und internationaler Ebene.
Der Bewertungsprozess hat inzwischen ein fortgeschrittenes Stadium erreicht; der EDSB hat am 7.März 2012 eine umfassende Stellungnahme ${ }^{1}$ zu den Gesetzgebungsvorschlägen herausgegeben, den Bewertungsprozess genau verfolgt und seine Beraterrolle im Laufe von 2012 erfüllt, indem er in den angemessenen Phasen und in den angemessenen Foren eingriff. Der EDSB wird die Entwicklungen weiterhin verfolgen und sieht eine fortlaufende und rechtzeitige Einbeziehung hierin und in verwandten bevorstehenden Initiativen wie etwa zum Datenschutz in der elektronischen Kommunikation (ePrivacy) vor.

Der EDSB verpflichtet sich, der Analyse dieser strategischen Bereiche 2013 umfangreiche Mittel zu widmen und wird zusätzlich eine Anzahl von (nicht oder weniger strategischen) Initiativen überwachen, die nichtsdestotrotz für den Datenschutz relevant sein können.

Eine der Pflichten des EDSB ist es nach der Verordnung (EG) Nr. 45/2001, die Europäische Kommission, das Europäische Parlament und den Rat zu Vorschlägen für die neue Gesetzgebung und einem breiten Spektrum anderer Fragen, die Auswirkungen auf den Datenschutz haben, zu beraten.

Der Europäische Datenschutzbeauftragte (EDSB) ist eine unabhängige Behörde, deren Aufgabe es ist, dafür zu sorgen, dass der Schutz personenbezogener Daten und der Privatsphäre gewährleistet ist und bewährte Verfahren in den Organen und Einrichtungen der EU gefördert werden. Er erfüllt diese Aufgabe, indem er

- die Verarbeitung personenbezogener Daten durch die EU-Verwaltung überwacht,

- in Bezug auf politische Maßnahmen und Rechtsvorschriften, die sich auf den Schutz der Privatsphäre auswirken, beratend tätig ist und

- mit vergleichbaren Behörden zusammenarbeitet, um einen kohärenten Datenschutz sicherzustellen.

\section{Der Europäische Datenschutzbeauftragte Peter Hustinx}

$1 \mathrm{http} / /$ www.edps.europa.eu/EDPSWEB/webdav/site/mySite/shared/ Documents/Consultation/Opinions/2012/12-03-07_EDPS_Reform_package_EN.pdf 